\title{
A proposal to measure the hadronic contribution to the $g-2$ in the space-like region *
}

\author{
Luca Trentadue $e^{1,2, \star \star}$ \\ 1 Università degli Studi di Parma, Dipartimento di Fisica e Scienze della Terra " Macedonio Melloni " \\ ${ }^{2}$ INFN, Sezione di Milano Bicocca, Milano, Italy
}

\begin{abstract}
We propose a novel approach to determine the leading hadronic corrections to the muon $g$-2. It consists in a measurement of the effective electromagnetic coupling in the space-like region. This method may become feasible at flavor factories as well as with $\mu$ e scattering resulting in a determination potentially competitive with the dispersive approach via time-like data.
\end{abstract}

\section{Introduction}

The motivation of this work [1,2] is due to a long-standing discrepancy between experiment and the Standard Model (SM) prediction for $a_{\mu}$, the muon anomalous magnetic moment. For this reason the hadronic corrections have been kept under close scrutiny [3-6]. The hadronic contribution represents the largest uncertainty of the Standard Model (SM) value and is comparable with the experimental one. When the new results from the $g$ - 2 experiments at Fermilab and J-PARC will reach the unprecedented precision of 0.14 parts per million (or better) [7-9], the uncertainty of the hadronic corrections will become the main limitation of this formidable test of the SM.

Vacuum Polarization (VP) makes $\alpha_{e m}$ running assuming a well defined effective value at any scale. Vacuum polarization and the effective charge are defined by:

$$
e^{2} \rightarrow e^{2}\left(q^{2}\right)=\frac{e^{2}}{1+\left(\Pi\left(q^{2}\right)-\Pi(0)\right)}
$$

and

$$
\alpha\left(q^{2}\right)=\frac{\alpha(0)}{1-\Delta \alpha} \Delta \alpha=-\operatorname{Re}\left(\Pi\left(q^{2}\right)-\Pi(0)\right) .
$$

$\Delta \alpha$ takes contributions from leptonic and hadronic elementary states among these the non-perturbative $\Delta \alpha_{\text {had }}$

$$
\Delta \alpha=\Delta \alpha_{\text {lept }}+\Delta \alpha_{\text {had }}+\Delta \alpha_{t o p}
$$

\footnotetext{
${ }^{\star}$ Work done in collaboration with: G. Abbiendi, C. Carloni Calame, U. Marconi, C. Matteuzzi, G. Montagna, O. Nicrosini, M. Passera, F. Piccinini, R. Tenchini and G. Venanzoni.

$\star \star$ e-mail: luca.trentadue@unipr.it
} 


$$
\Delta \alpha_{h a d}^{(5)}\left(M_{z}^{2}\right)=-\frac{\alpha M_{z}^{2}}{3 \pi} \operatorname{Re} \int_{4 m_{\pi}^{2}}^{\infty} d s \frac{R(s)}{s\left(s-M_{z}^{2}-i \epsilon\right)}
$$

A direct measurement of the running of $\alpha_{e m}(s / t)$ in space/time-like regions can show the running of $\alpha_{e m}$. It can provide a test of duality far away form resonances and ita has been done in the past by few experiments at $e^{+} e^{-}$colliders by comparing well known QED processes with some reference ( either data or MonteCarlo ) normalization:

$$
\left(\frac{\alpha\left(q^{2}\right)}{\alpha\left(q_{0}^{2}\right)}\right)^{2} \simeq \frac{N_{\text {signal }}\left(q^{2}\right)}{N_{\text {norm }}\left(q_{0}^{2}\right)} .
$$

$N_{\text {signal }}$ can be any QED process as muon pairs production or others similar ones and $N_{\text {norm }}$ can be the Bhabha process, a pure QED channel as $\gamma \gamma$ pair production as well as theory, or any other reference process. $q_{0}$ represents a scale chosen as starting point for the running.

\section{$2 a_{m u}^{H L O}$ calculation}

The leading-order hadronic contribution to the muon $g$ - 2 is given by the well-known formula $[6,13]$

$$
a_{\mu}^{\mathrm{HLO}}=\frac{\alpha}{\pi^{2}} \int_{0}^{\infty} \frac{d s}{s} K(s) \operatorname{Im} \Pi_{\mathrm{had}}(s+i \epsilon),
$$

where $\Pi_{\text {had }}(s)$ is the hadronic part of the photon vacuum polarization, $\epsilon>0$, and

$$
K(s)=\int_{0}^{1} d x \frac{x^{2}(1-x)}{x^{2}+(1-x)\left(s / m_{\mu}^{2}\right)}
$$

is a positive kernel function with $m_{\mu}$ the muon mass.

As the total cross section for hadron production in low-energy $e^{+} e^{-}$annihilations is related to the imaginary part of $\Pi_{\text {had }}(s)$ via the optical theorem, the dispersion integral in eq. (6) is computed integrating experimental time-like $(s>0)$ data up to a certain value of $s[4,17,18]$. The high-energy tail of the integral is calculated by using perturbative QCD [19]. In a recent work the KLOE collaboration has obtained $[21,22]$ the the first measurement of the running of the effective QED coupling constant $\alpha(s)$ in the time-like region $0.6<s<0.975 \mathrm{GeV}$ at DAФNE by using the Initial State Radiation approach [20] in the process $e^{+} e^{-} \rightarrow \mu^{+} \mu^{-} \gamma$.

Alternatively, if we exchange the $x$ and $s$ integrations in eq. (6) we obtain [23]

$$
a_{\mu}^{\mathrm{HLO}}=\frac{\alpha}{\pi} \int_{0}^{1} d x(x-1) \bar{\Pi}_{\mathrm{had}}[t(x)],
$$

where $\bar{\Pi}_{\text {had }}(t)=\Pi_{\text {had }}(t)-\Pi_{\text {had }}(0)$ and

$$
t(x)=\frac{x^{2} m_{\mu}^{2}}{x-1}<0
$$

is a space-like squared four-momentum. If we invert eq. (9), we get $x=(1-\beta)\left(t / 2 m_{\mu}^{2}\right)$, with $\beta=$ $\left(1-4 m_{\mu}^{2} / t\right)^{1 / 2}$, and from eq. (8) we obtain

$$
a_{\mu}^{\mathrm{HLO}}=\frac{\alpha}{\pi} \int_{-\infty}^{0} \bar{\Pi}_{\text {had }}(t)\left(\frac{\beta-1}{\beta+1}\right)^{2} \frac{d t}{t \beta} .
$$


Equation (10) has been used for lattice QCD calculations of $a_{\mu}^{\text {HLO }}$ [24]; while the results are not yet competitive with those obtained with the dispersive approach via time-like data, their errors are expected to decrease significantly in the next few years [25].

The effective fine-structure constant at squared momentum transfer $q^{2}$ is defined above and is $\Delta \alpha\left(q^{2}\right)=-\operatorname{Re} \bar{\Pi}\left(q^{2}\right)$. The purely leptonic part, $\Delta \alpha_{\text {lep }}\left(q^{2}\right)$, can be calculated order-by-order in perturbation theory - it is known up to three loops in QED [26] (and up to four loops in specific $q^{2}$ limits [27]). As $\operatorname{Im} \bar{\Pi}\left(q^{2}\right)=0$ for negative $q^{2}$, eq. (8) can be rewritten in the form [28]

$$
a_{\mu}^{\mathrm{HLO}}=\frac{\alpha}{\pi} \int_{0}^{1} d x(1-x) \Delta \alpha_{\mathrm{had}}[t(x)]
$$

Equation (11), involving the hadronic contribution to the running of the effective fine-structure constant at space-like momenta, can be further formulated in terms of the Adler function [29], defined as the logarithmic derivative of the vacuum polarization, which, in turn, can be calculated via a dispersion relation with time-like hadroproduction data and perturbative QCD [28, 30].

We are going to proceed differently, proposing to calculate eq. (11) by measurements of the effective electromagnetic coupling in the space-like region. The hadronic contribution to the running of $\alpha$ in the space-like region, $\Delta \alpha_{\text {had }}(t)$ (see eq. (3)), can be extracted by comparing Bhabha scattering data with Monte Carlo predictions. The Leading Order (LO) Bhabha cross section receives contributions from $t$ - and $s$-channel photon exchange amplitudes. At Next to Leading Order (NLO) in QED, it is customary to distinguish corrections with an additional virtual photon or the emission of a real photon (photonic NLO) from those originated by the insertion of the vacuum polarization corrections into the LO photon propagator.

Let us consider a few simple points. In fig. 1 (left) we plot the integrand $(1-x) \Delta \alpha_{\text {had }}[t(x)]$ of eq. (11) using the output of the routine hadr5n12 [33] (which uses time-like hadroproduction data and perturbative QCD). The range $x \in(0,1)$ corresponds to $t \in(-\infty, 0)$, with $x=0$ for $t=0$. The peak of the integrand occurs at $x_{\text {peak }} \simeq 0.914$ where $t_{\text {peak }} \simeq-0.108 \mathrm{GeV}^{2}$ and $\Delta \alpha_{\text {had }}\left(t_{\text {peak }}\right) \simeq 7.86 \times 10^{-4}$ (see fig. 1 (right)). Such relatively low $t$ values can be explored at $e^{+} e^{-}$colliders with center-of-mass energy $\sqrt{s}$ around or below $10 \mathrm{GeV}$ where

$$
t=-\frac{s}{2}(1-\cos \theta)\left(1-\frac{4 m_{e}^{2}}{s}\right),
$$

$\theta$ is the electron scattering angle and $m_{e}$ is the electron mass. Depending on $s$ and $\theta$, the integrand of eq. (11) can be measured in the range $x \in\left[x_{\min }, x_{\max }\right]$, as shown in fig. 2 (left). Note that to span low $x$ intervals, larger $\theta$ ranges are needed as the collider energy decreases. In this respect, $\sqrt{s} \sim 3 \mathrm{GeV}$ appears to be very convenient, as an $x$ interval $[0.30,0.98]$ can be measured varying $\theta$ between $\sim 2^{\circ}$ and $28^{\circ}$. It is also worth remarking that data collected at flavor factories, such as DAФNE (Frascati), VEPP-2000 (Novosibirsk), BEPC-II (Beijing), PEP-II (SLAC) and SuperKEKB (Tsukuba), and possibly at a future high-energy $e^{+} e^{-}$collider, like FCC-ee (TLEP) [34] or ILC [35], can help to cover different and complementary $x$ regions.

Furthermore, given the smoothness of the integrand, values outside the measured $x$ interval may be interpolated with some theoretical input. In particular, the region below $x_{\min }$ will provide a relatively small contribution to $a_{\mu}^{\text {HLO }}$, while the region above $x_{\max }$ may be obtained by extrapolating the curve from $x_{\max }$ to $x=1$, where the integrand is null, or using perturbative QCD.

The analytic dependence of the MC Bhabha predictions on $\alpha(t)$ (and, in turn, on $\Delta \alpha_{\text {had }}(t)$ ) is not trivial, and a numerical procedure has to be devised to extract it from the data.This was not the case 

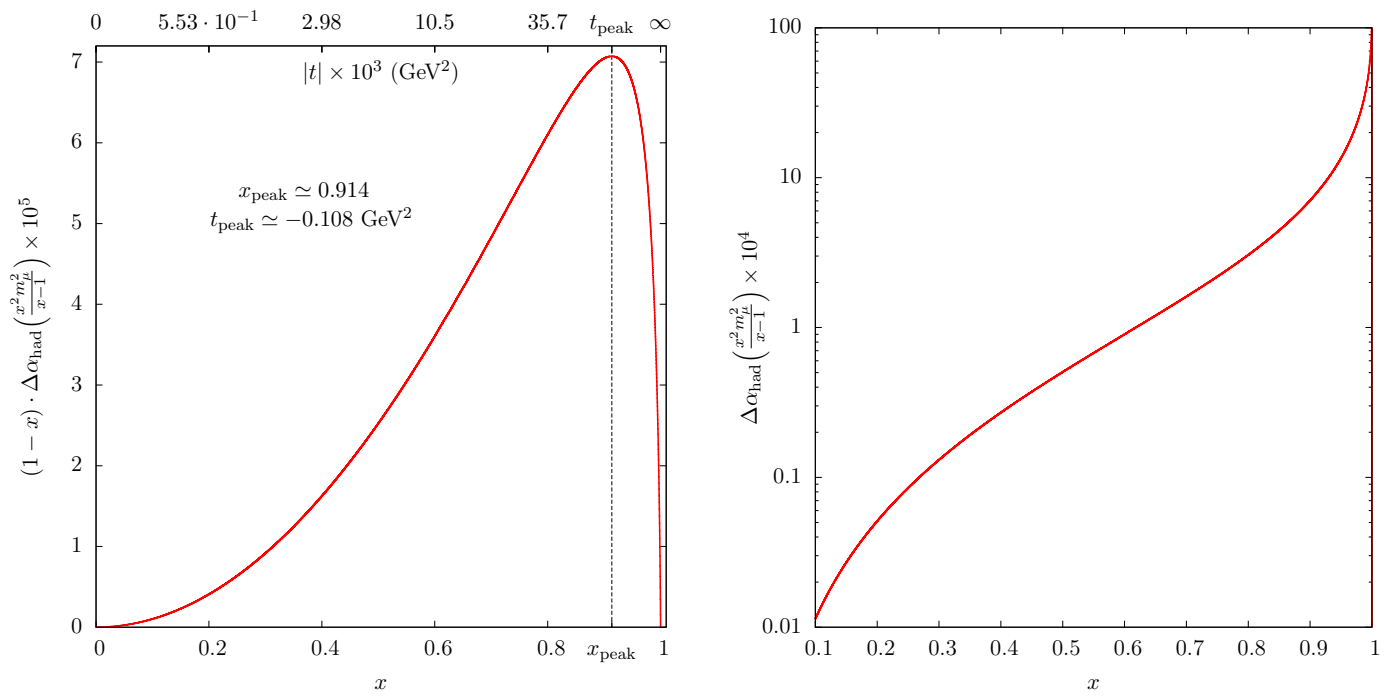

Figure 1. Left: The integrand $(1-x) \Delta \alpha_{\text {had }}[t(x)] \times 10^{5}$ as a function of $x$ and $t$. Right: $\Delta \alpha_{\text {had }}[t(x)] \times 10^{4}$.
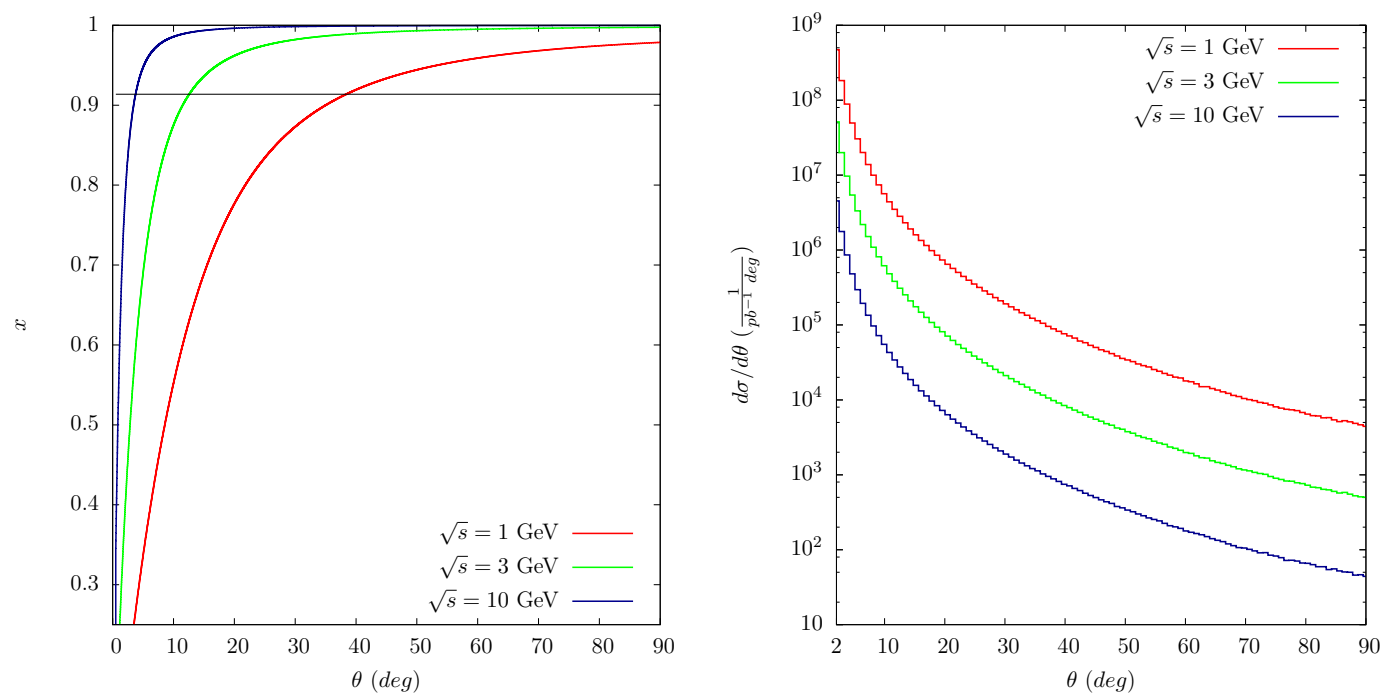

Figure 2. Left: Ranges of $x$ values as a function of the electron scattering angle $\theta$ for three different center-ofmass energies. The horizontal line corresponds to $x=x_{\text {peak }} \simeq 0.914$. Right: Bhabha differential cross section obtained with BabaYaga [31] as a function of $\theta$ for the same three values of $\sqrt{s}$ in the angular range $2^{\circ}<\theta<90^{\circ}$. 
for example in refs. [14, 15]: there $\alpha(t)$ was extracted from Bhabha data in the very forward region at LEP, where the $t$ channel diagrams are by far dominant and $\alpha(t)$ factorizes (see for example [16]). In formulae, we have to find a function $\alpha(t)$ such that

$$
\left.\frac{d \sigma}{d t}\right|_{\mathrm{data}}=\left.\frac{d \sigma}{d t}(\alpha(t), \alpha(s))\right|_{\mathrm{MC}},
$$

where we explicitly kept apart the dependence on the time-like VP $\alpha(s)$ because we are only interested in $\alpha(t)$. We emphasise that, in our analysis, $\alpha(s)$ is an input parameter. Being the Bhabha cross section in the forward region dominated by the $t$-channel exchange diagrams, we checked that the present $\alpha(s)$ uncertainty induces in this region a relative error on the $\theta$ distribution of less than $\sim 10^{-4}$ (which is part of the systematic error).

We propose to perform the numerical extraction of $\Delta \alpha_{\text {had }}(t)$ from the Bhabha distribution of the $t$ Mandelstam variable. The idea is to let $\alpha(t)$ vary in the MC sample around a reference value and choose, bin by bin in the $t$ distribution, the value that minimizes the difference with data. The procedure can be sketched as follows:

1. choose a reference function returning the value of $\Delta \alpha_{\text {had }}(t)$ (and hence $\alpha(t)$ ) to be used in the MC sample, we call it $\bar{\alpha}(t)$;

2. for each generated event, calculate $N$ MC weights by rescaling $\bar{\alpha}(t) \rightarrow \bar{\alpha}(t)+\frac{i}{N} \delta(t)$, where $i \in[-N, N]$ and $\delta(t)$ is for example the error induced on $\bar{\alpha}(t)$ by the error on $\Delta \alpha_{\text {had }}(t)$. Being done on an event by event basis, the full dependence on $\alpha(t)$ of the MC differential cross section can be kept;

3. for each bin $j$ of the $t$ distribution, compare the experimental differential cross section with the MC predictions and choose the $i_{j}$ which minimizes the difference;

4. $\bar{\alpha}\left(t_{j}\right)+\frac{i_{j}}{N} \delta\left(t_{j}\right)$ will be the extracted value of $\alpha\left(t_{j}\right)$ from data in the $j^{t h}$ bin. $\Delta \alpha_{\text {had }}\left(t_{j}\right)$ can then be obtained through the relation between $\alpha(t)$ and $\Delta \alpha_{\text {had }}(t)$.

We finally find, for each bin $j$ of the $t$ distribution,

$$
\left.\frac{d \sigma}{d t}\right|_{j, \text { data }}=\left.\frac{d \sigma}{d t}\left(\bar{\alpha}(t)+\frac{i_{j}}{N} \delta(t), \alpha(s)\right)\right|_{j, \mathrm{MC}} .
$$

We remark that the algorithm does not assume any simple dependence of the cross section on $\alpha(t)$, which can in fact be general, mixing $s, t$ channels and higher order radiative corrections, relevant (or not) in different $t$ domains.

In order to test our procedure, we perform a pseudo-experiment: we generate pseudo-data using the parameterization $\Delta \alpha_{\text {had }}^{I}(t)$ of refs. $[18,36]$ and check if we can recover it by inserting in the MC the (independent) parameterization $\Delta \alpha_{\text {had }}^{I I}(t)$ (corresponding to $\bar{\alpha}(t)$ of eq. 14) of ref. [33] by means of the method described above. For this exercise, we use the generator BabaYaga in its most complete setup, generating events at $\sqrt{s}=1.02 \mathrm{GeV}$, requiring $10^{\circ}<\theta_{ \pm}<170^{\circ}, E_{ \pm}>0.4 \mathrm{GeV}$ and an acollinearity cut of $15^{\circ}$. We choose $\delta(t)$ to be the error induced on $\alpha(t)$ by the $1-\sigma$ error on $\Delta \alpha_{\text {had }}(t)$, which is returned by the routine of ref. [33], we set $N=150$, and we produce distributions with 200 bins. We note that in the present exercise $\alpha(s)$ and all the radiative corrections both in the pseudo-data and in the MC samples are exactly the same, because we are interested in testing the algorithm rather than assessing the achievable accuracy, at least at this stage.

In fig. 3, $\Delta \alpha_{\text {had }}^{\text {extr }}$ is the result extracted with our algorithm, corresponding to the minimizing set of $i_{j}$ : the figure shows that our method is capable of recovering the underlying function $\Delta \alpha_{\text {had }}(t)$ inserted 
into the "data". As the difference between $\Delta \alpha_{\text {had }}^{I}$ and $\Delta \alpha_{\text {had }}^{\text {extr }}$ is hardly visible on an absolute scale, in fig. 3 all the functions have been divided by $\Delta \alpha_{\text {had }}^{I I}$ to display better the comparison between $\Delta \alpha_{\text {had }}^{I}$ and $\Delta \alpha_{\text {had }}^{\text {extr }}$.

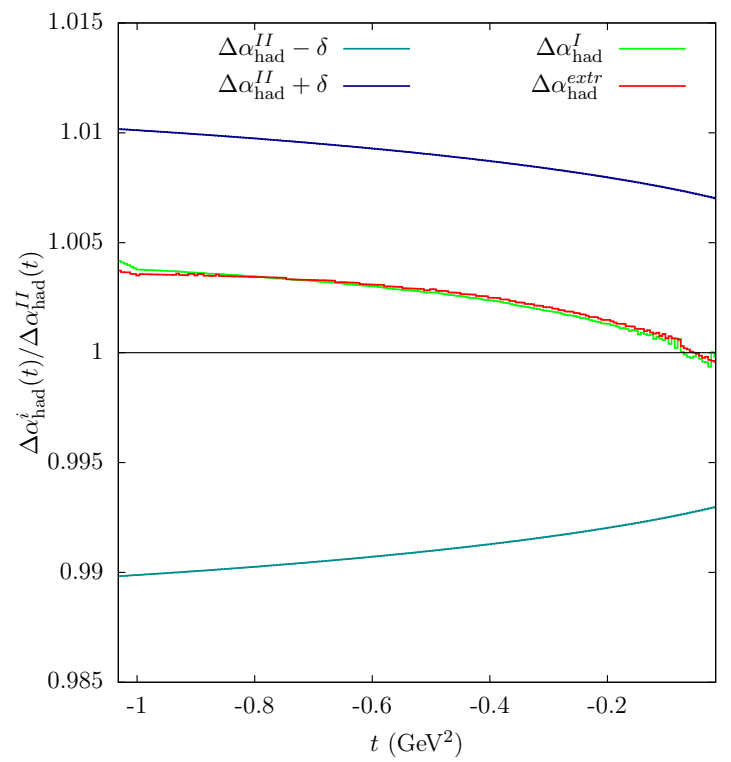

Figure 3. The extracted function $\Delta \alpha_{\text {had }}^{\text {extr }}(t)$ compared to the function $\Delta \alpha_{\text {had }}^{I}(t)$ used in the pseudo-data (see text). The functions $\Delta \alpha_{\text {had }}^{I I}(t) \pm \delta(t)$ are shown to display the range spanned by the MC samples. All functions have been divided by $\Delta \alpha_{\text {had }}^{I I}(t)$. The tiny difference between $\Delta \alpha_{\text {had }}^{I}$ and $\Delta \alpha_{\text {had }}^{\text {extr }}$ is due to the binning discretization.

In order to assess the achievable accuracy on $\Delta \alpha_{\text {had }}(t)$ with the proposed method, we remark that the LO contribution to the cross section is quadratic in $\alpha(t)$, thus we have

$$
\frac{1}{2} \frac{\delta \sigma}{\sigma} \simeq \frac{\delta \alpha}{\alpha} \simeq \delta \Delta \alpha_{\mathrm{had}}
$$

Equation (15) relates the absolute error on $\Delta \alpha_{\text {had }}$ with the relative error on the Bhabha cross section. Let us stress here that:

1. From the theoretical point of view, the present accuracy of the MC predictions [32] is at the level of about $0.05 \%$, which implies that the precision that our method can, at best, set on $\Delta \alpha_{\text {had }}(t)$ is $\delta \Delta \alpha_{\text {had }}(t) \simeq 2 \cdot 10^{-4}$. Any further improvement requires the inclusion of the NNLO QED corrections into the MC codes ( see Ref. [32] ).

2. Experimentally a measurement of $a_{\mu}^{\mathrm{HLO}}$ from space-like data competitive with the current time-like evaluations would require an $O(1 \%)$ accuracy. Statistical considerations show that a $3 \%$ fractional accuracy on the $a_{\mu}^{\mathrm{HLO}}$ integral can be obtained by sampling the integrand $(1-x) \Delta \alpha_{\text {had }}[t(x)]$ in $\sim 10$ points around the $x$ peak with a fractional accuracy of $10 \%$. Given the value of $O\left(10^{-3}\right)$ for $\Delta \alpha_{\text {had }}$ at $x=x_{\text {peak }}$, this implies that the cross section must be known with relative accuracy of $\sim 2 \times 10^{-4}$. Such a statistical accuracy, although challenging, can be obtained at flavor factories, as shown in fig. 2 (right). With an integrated luminosity of $O(1)$, 
$O(10), O(100) \mathrm{fb}^{-1}$ at $\sqrt{s}=1,3$ and $10 \mathrm{GeV}$, respectively, the angular region of interest can be covered with a $0.01 \%$ accuracy per degree. The experimental systematic error must match the same level of accuracy.

A source of experimental systematic errors comes from the machine luminosity, which can be normalized by calculating a theoretical cross section in principle not depending on $\Delta \alpha_{\text {had }}$. We devise two possible options for the normalization process:

1. To use the $e^{+} e^{-} \rightarrow \gamma \gamma$ process, which has no dependence on $\Delta \alpha_{\text {had }}$, at least up to NNLO order;

2. To use the Bhabha process at $t \sim 10^{-3} \mathrm{GeV}^{2}(x \sim 0.3)$, where the dependence on $\Delta \alpha_{\text {had }}$ is of $O\left(10^{-5}\right)$ and can be safely neglected.

It is worth quoting that a detailed analysis of the systematic errors involved in the measurement of the luminosity has been carried out at LEP by the OPAL collaboration reaching the final accuracy of $O\left(10^{-4}\right)[15,37]$.

\section{An alternative approach}

Always within the space-like approach, an alternative possibility has been investigated [2]. It consists in using a fixed target $\mu \rightarrow e$ scattering process and to analyse the elastic muon distribution in the forward region. A new experiment has to be devised to measure the running of the fine-structure constant in the space-like region by scattering high-energy muons on atomic electrons of a low- $Z$ target through the process $\mu e \rightarrow \mu e$. The differential cross section of this process, measured as a function of the squared momentum transfer $t=q^{2}<0$, provides direct sensitivity to the leading-order hadronic contribution to the muon anomaly $a_{\mu}^{\mathrm{HLO}}$. By using a muon beam of $150 \mathrm{GeV}$, with an average rate of $\sim 1.3 \times 10^{7} \mathrm{muon} / \mathrm{s}$, currently available at the CERN North Area, a statistical uncertainty of $\sim 0.3 \%$ can be achieved on $a_{\mu}^{\mathrm{HLO}}$ after two years of data taking. As the Bhabha process also this direct measurement of $a_{\mu}^{\mathrm{HLO}}$ will provide an independent determination, potentially competitive with the time-like dispersive approach, to consolidate, with a firmer interpretation of the measurements of the future muon $g-2$ experiments, the theoretical prediction for the muon $g-2$ in the Standard Model.

A series of preliminary considerations on the detector have been discussed [2].

In order to perform the planned measurement to the required precision, a dedicated detector is necessary. We describe here a possible setup to measure the following observables:

- direction and momentum of the incident muon;

- directions of the outgoing electron and muon.

The CERN muon beam M2, used at $150 \mathrm{GeV}$, has the characteristics needed for such a measurement. The beam intensity appears to be adequate to provide the required event yield. The beam time structure allows to tag the incident muon while keeping low the background related to incoming particles (e.g. electrons). The electrons contamination is very small. The beam provides both positive and negative muons, which we plan to use.

The target consists of atomic electrons. To reach the required statistics, the target must consist of an adequate amount of material to give a sufficient number of electron scattering centres. The target has to be made of a low- $Z$ material to minimize the impact of multiple scattering and the background due to bremsstrahlung and pair production processes. A promising idea for the detector is to use 20 layers of $\mathrm{Be}$ (or $\mathrm{C}$ ) coupled to Si planes, spaced by intermediate air gaps, located at a relative distance of one meter from each other. Fig. 4 shows the basic layout, presently under study. The 

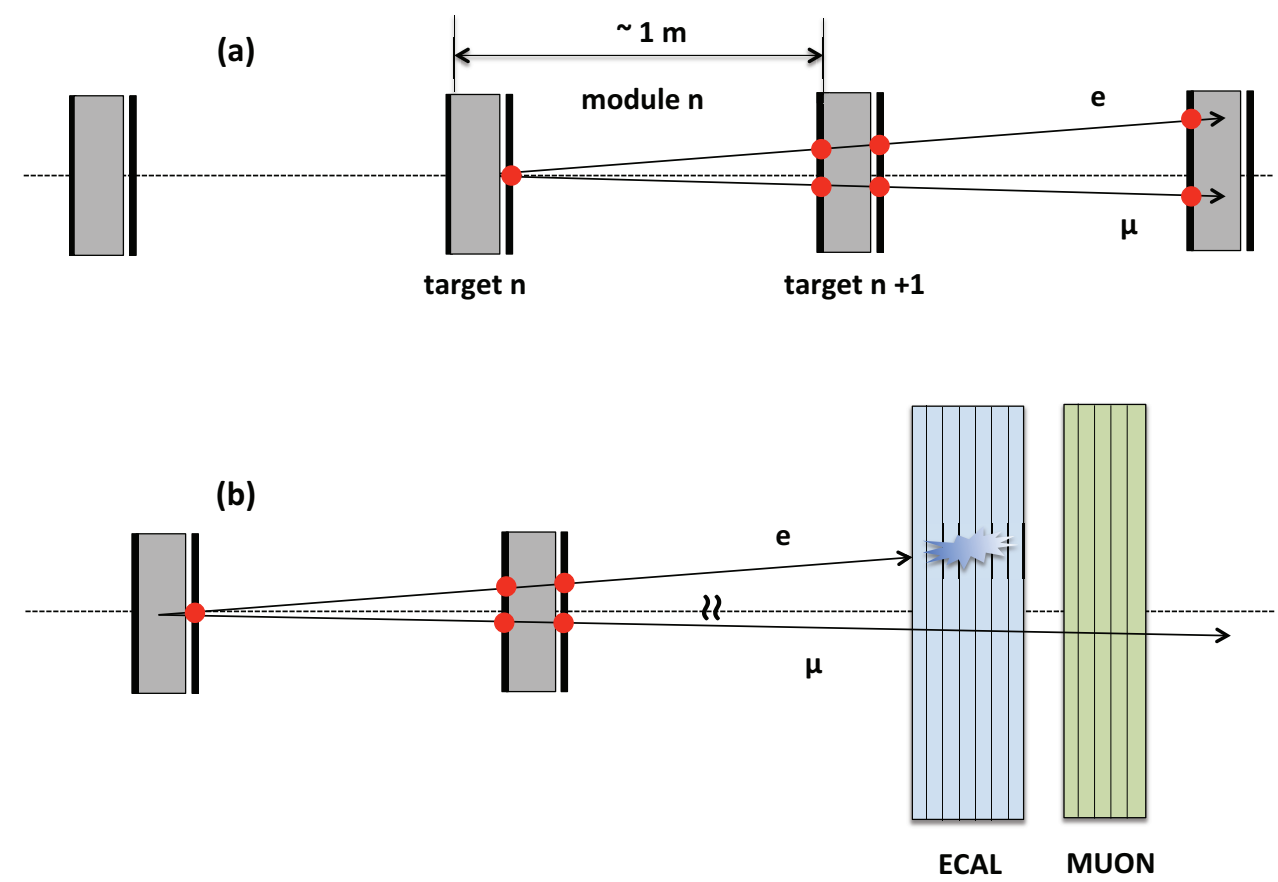

Figure 4. Scheme of a possible detector layout. (a) The detector is a modular system. Each module consists of a low- $Z$ target (Be or $\mathrm{C}$ ) and two silicon tracking stations located at a distance of one meter. (b) To perform the $\mu / e$ discrimination in the case of small scattering angles (both $\theta_{\mu}$ and $\theta_{e}$ below $5 \mathrm{mrad}$ ) the detector is equipped with an electromagnetic calorimeter and a muon detector.

arrangement provides both a distributed target with low- $Z$ and the tracking system. As downstream particle identifiers we plan to use a calorimeter for the electrons and a muon system for the muons (a filter plus active planes). This particle identifier system is required to solve the muon-electron ambiguity for electron scattering angles around (2-3) $\operatorname{mrad}(c f$. Fig. 5). The preliminary studies of such an apparatus, performed by using GEANT4, indicate an angular resolution for the outgoing particles of $\sim 0.02 \mathrm{mrad}$.

The detector acceptance must cover the region of the signal, with the electron emitted at extremely forward angles and high energies, as well as the normalization region, where the electron has much lower energy (around $1 \mathrm{GeV}$ ) and an emission angle of some tens of mrad. The boosted kinematics of the process allows the detector to cover almost $100 \%$ of the acceptance.

The incoming muons have to be tagged and their direction and momentum precisely measured. To this purpose, a detector similar to those used by COMPASS [39] or NA62 [40] can be employed.

- the angle of the scattered electron and muon are correlated as shown in Fig. 5 (for muons of $150 \mathrm{GeV}$ ). This constraint is extremely important to select elastic scattering events, rejecting back- 
ground events from radiative or inelastic processes and to minimize systematic effects in the determination of $t$. Note that for scattering angles of (2-3) mrad there can be an ambiguity between the outgoing electron and muon, as their angles and momenta are similar. To associate them correctly it is necessary to identify the two particles by means of downstream dedicated detectors (calorimeter and muon detectors).

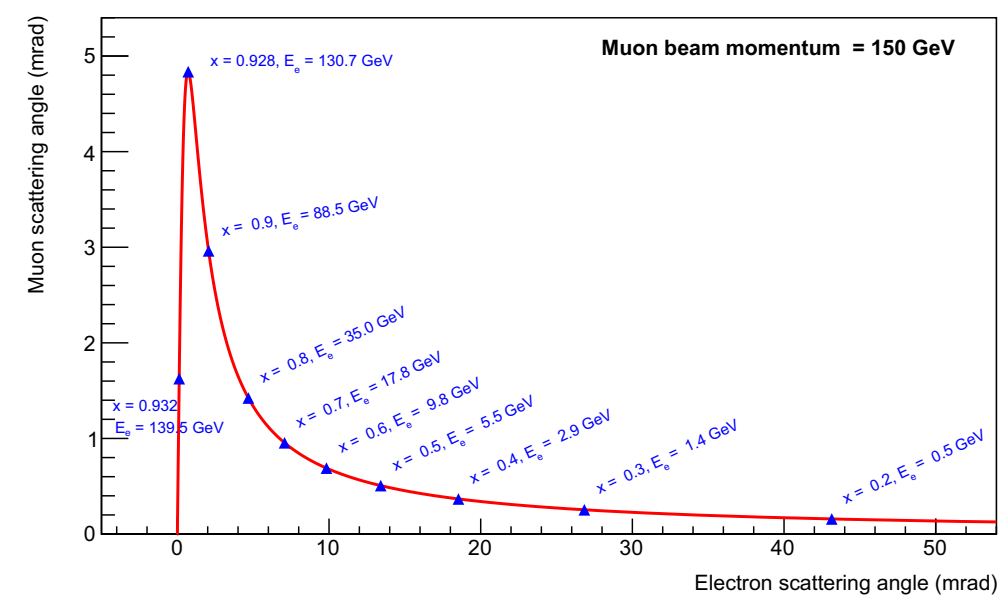

Figure 5. The relation between the muon and electron scattering angles for $150 \mathrm{GeV}$ incident muon momentum.

We estimate the statistical sensitivity of this experiment (supplemented with large $|t|$ contributions derived from pQCD) to be $\sim 0.3 \%$ on the value of $a_{\mu}^{\mathrm{HLO}}$ after two years of data taking, using 30 experimental points in $x$, assuming a running time of $2 \times 10^{7} \mathrm{~s} / \mathrm{yr}$, and a muon beam of $150 \mathrm{GeV}$ with an average intensity of $\sim 1.3 \times 10^{7} \mathrm{muon} / \mathrm{s}$ as the beam currently available at the CERN North Area [38].

A series of systematic uncertainties should be taken into account.

Significant contributions of the hadronic vacuum polarization to the $\mu e \rightarrow \mu e$ differential cross section are essentially restricted to electron scattering angles below $10 \mathrm{mrad}$, corresponding to electron energies above $10 \mathrm{GeV}$. The net effect of these contributions is to increase the cross section by a few per mille: a precise determination of $a_{\mu}^{\mathrm{HLO}}$ requires not only high statistics, but also a high systematic accuracy, as the final goal of the experiment is equivalent to a determination of the differential cross section with $\sim 10$ ppm systematic uncertainty at the peak of the integrand function ( $c f$. Fig. 1).

Such an accuracy can be achieved if the efficiency is kept highly uniform over the entire $q^{2}$ range, including the normalization region, and over all the detector components. This motivates the choice of a purely angular measurement: an acceptance of tens of mrad can be covered with a single sensor of modern silicon detectors, positioned at a distance of about one meter from the target. It has to be stressed that particle identification (electromagnetic calorimeter and muon filter) is necessary to solve the electron-muon ambiguity in the region below $5 \mathrm{mrad}$. The wrong assignment probability can be measured with the data by using the rate of muon-muon and electron-electron events.

Another requirement for reaching very high accuracy is to measure all the relevant contributions to systematic uncertainties from the data themselves. An important effect, which distinguishes the normalization from the signal region, is multiple scattering, as the electron energy in this region is 
as low as $1 \mathrm{GeV}$. Multiple scattering breaks the muon-electron two-body angular correlation, moving events out of the kinematic line in the 2D plot of Fig. 5. In addition, multiple scattering in general causes acoplanarity, while two-body events are planar, within resolution. These facts allow multiple scattering effects to be modelled and measured by using data. An additional handle on multiple scattering could be the inclusion of a thin layer in the apparatus, made of the same material as the main target modules. This possibility will be studied in detail with dedicated simulations.

In experiments dedicated to high-precision measurements, several systematic effects can be explored within the experiment itself. In this respect the proposed modularity of the apparatus will help. A test with a single module could provide a proof-of-concept of the proposed methods.

From the theoretical point of view, the control of the systematic uncertainties requires the development of high-precision Monte Carlo tools, including the relevant radiative corrections to reach the needed theoretical precision. To this aim, QED radiative corrections at leading-logarithmic level resummed at all orders of perturbation theory and matched to the exact $O(\alpha)$ correction (as for instance implemented in the BabaYaga event generator [31] for Bhabha scattering) are mandatory, ensuring a theoretical precision at the level of $O\left(10^{-4}\right)$ on the differential cross section. Moreover, by using the ratio of the cross sections in the signal and normalization regions, we expect that the theoretical uncertainties will be further reduced to the level of $O\left(10^{-5}\right)$, due to partial cancellation of common radiative corrections. Work is in progress to extend BabaYaga to $\mu e \rightarrow \mu e$ scattering and to quantify the actual accuracy on the computation of the ratio of cross sections by means of dedicated Monte Carlo simulations. Any further improvement in the theoretical accuracy would require the matching of QED resummation with exact two-loop corrections, which are not available at present for the $\mu e \rightarrow \mu e$ process but are within reach.

\section{Conclusions}

We presented a novel approach to determine the leading hadronic correction to the muon $g$ - 2 based on measurements of the running of $\alpha(t)$ in the space-like region. An alternative formula for $a_{\mu}^{H L O}$ in the space-like region has been studied in detail. It emphasizes low values of $t \leq 1 \mathrm{GeV}^{2}$ which can be explored at low and at high energies machines.

We have also argued that this requires a measurement of the Bhabha cross section as of the $\mu \rightarrow e$ elastic distribution at relatively small angles, with an accuracy of the order $10^{-4}$ or better.

The $\mu \rightarrow e$ experiment is primarily based on a precise measurement of the scattering angles of the two outgoing particles as the $q^{2}$ of the muon-electron interaction can be directly determined by the electron (or muon) scattering angle.

The normalization of the cross section is provided by the very same $\mu e \rightarrow \mu e$ process in the low- $q^{2}$ region, where the effect of the hadronic corrections on the fine-structure constant is negligible. Such a simple and robust technique has the potential to keep systematic effects under control, aiming to reach a systematic uncertainty of the same order as the statistical one. For this purpose a preliminary detector layout has been described. By considering a beam of $150 \mathrm{GeV}$ muons with an average intensity of $\sim 1.3 \times 10^{7}$ muons/s, a statistical uncertainty of $\sim 0.3 \%$ can be achieved on $a_{\mu}^{\mathrm{HLO}}$ in two years of data taking.

We have shown that an advantage of the muon beam is the possibility of employing a modular apparatus, with the target subdivided in subsequent layers. A low- $Z$ solid target is preferred in 
order to provide the required event rate, limiting at the same time the effect of multiple scattering as well as of other types of muon interactions (pair production, bremsstrahlung and nuclear interactions).

We have shown that this approach, even if challenging, may become feasible both by using data collected at present flavor factories and at future high-energy $e^{+} e^{-}$colliders as well as with fixed target $\mu \rightarrow e$ elastic scattering.

The proposed determinations can become competitive with the accuracy of the present results obtained with the dispersive approach via time-like data. Reaching such an accuracy demands dedicated experiments and theoretical work for the next few years.

We propose a new approach to evaluate the leading-order hadronic contribution to the muon anomaly. The direct measurement of the hadronic contribution to anomalous muon magnetic moment will provide an independent determination, competitive with the time-like dispersive approach, and consolidate the theoretical prediction for the muon g-2 in the Standard Model. It will allow a firmer interpretation of the measurements of the future muon g- 2 experiments at Fermilab and J-PARC.

This new approach may become a path within an unexplored region of the field theoretical dynamics. It may lead to a possibly long series of phenomenological results. The (crossed) t-channel dynamics, as complementary and independent with respect to the s-channel one, will permit an alternative new approach to the Standard Model precision physics.

\section{Acknowledgements}

I wish to thank Antonio Di Domenico for organizing and for involving me in this lively Workshop.

\section{References}

[1] C. Carloni Calame, M. Passera, G. Venanzoni, L. Trentadue, Phys. Lett. B 746 (2015 ) 325-329.

[2] G. Abbiendi, C.M. Carloni Calame, U. Marconi, C. Matteuzzi, G. Montagna, O. Nicrosini M. Passera, F. Piccinini, R. Tenchini, L. Trentadue, G. Venanzoni, Eur. Phys. J. C 77 (2017) no.3, 139 doi:10.1140/epjc/s10052-017-4633-z [arXiv:1609.08987 [hep-ex]].

[3] G.W. Bennett et al. [Muon g-2 Collaboration], Phys. Rev. D 73 (2006) 072003.

[4] F. Jegerlehner, A. Nyffeler, Phys. Rept. 477 (2009) 1.

[5] T. Blum et al., arXiv:1311.2198 [hep-ph]; K. Melnikov, A. Vainshtein, Springer Tracts Mod. Phys. 216 (2006) 1; M. Davier, W.J. Marciano, Ann. Rev. Nucl. Part. Sci. 54 (2004) 115; M. Passera, J. Phys. G 31 (2005) R75; M. Knecht, Lect. Notes Phys. 629 (2004) 37.

[6] F. Jegerlehner, "The anomalous magnetic moment of the muon," Springer Tracts Mod. Phys. 226, 2008.

[7] J. Grange et al. [Muon g-2 Collaboration], arXiv:1501.06858 [physics.ins-det].

[8] G. Venanzoni [Muon g-2 Collaboration], arXiv:1411.2555 [physics.ins-det].

[9] N. Saito [J-PARC g-2/EDM Collaboration], AIP Conf. Proc. 1467 (2012) 45.

[10] G. Venanzoni, Nuovo Cim. C 037 (2014) 02, 165; G. Venanzoni, Frascati Phys. Ser. 54 (2012) 52.

[11] G.V. Fedotovich [CMD-2 Collaboration], Nucl. Phys. Proc. Suppl. 181-182 (2008) 146. 
[12] G. Colangelo, M. Hoferichter, A. Nyffeler, M. Passera, P. Stoffer, Phys. Lett. B 735 (2014) 90.

[13] C. Bouchiat, L. Michel, J. Phys. Radium 22 (1961) 121; L. Durand, Phys. Rev. 128 (1962) 441 [Erratum-ibid. 129 (1963) 2835]; M. Gourdin, E. De Rafael, Nucl. Phys. B 10 (1969) 667.

[14] A.B. Arbuzov, D. Haidt, C. Matteuzzi, M. Paganoni and L. Trentadue, Eur. Phys. J. C 34 (2004) 267.

[15] G. Abbiendi et al. [OPAL Collaboration], Eur. Phys. J. C 45 (2006) 1.

[16] A.B. Arbuzov, V.S. Fadin, E.A. Kuraev, L.N. Lipatov, N.P. Merenkov, L. Trentadue, Nucl. Phys. B485 (1997) 457-502;

A. B. Arbuzov, K. I. Gach, V. Y. Gonchar, E. A. Kuraev, N. P. Merenkov and L. Trentadue, Phys. Lett. B 399 (1997) 312;

A. B. Arbuzov, V. S. Fadin, E. A. Kuraev, L. N. Lipatov, N. P. Merenkov and L. G. Trentadue, Phys. Lett. B 394 (1997) 218;

A. B. Arbuzov, E. A. Kuraev, N. P. Merenkov and L. Trentadue, Phys. Atom. Nucl. 60 (1997) 591 [Yad. Fiz. 60N4 (1997) 673];

A. B. Arbuzov, E. A. Kuraev, N. P. Merenkov and L. Trentadue, J. Exp. Theor. Phys. 81 (1995) 638 [Zh. Eksp. Teor. Fiz. 108 (1995) 1164];

V. S. Fadin, E. A. Kuraev, L. Trentadue, L. N. Lipatov and N. P. Merenkov, Phys. Atom. Nucl. 56 (1993) 1537 [Yad. Fiz. 56N11 (1993) 145].

[17] M. Davier, A. Hoecker, B. Malaescu, Z. Zhang, Eur. Phys. J. C 71 (2011) 1515 [Erratum-ibid. C 72 (2012) 1874].

[18] K. Hagiwara, R. Liao, A.D. Martin, D. Nomura, T. Teubner, J. Phys. G 38 (2011) 085003.

[19] R.V. Harlander, M. Steinhauser, Comput. Phys. Commun. 153 (2003) 244.

[20] A. B. Arbuzov, E. A. Kuraev, N. P. Merenkov and L. Trentadue, JHEP 9812 (1998) 009.

[21] A. Anastasi et al. arXiv:1609.06631v1 [hep-ex] 21 Sep 2016, Phys.Lett. B767 (2017) 485-492.

[22] See the talk of G. Venanzoni at this Workshop.

[23] B.E. Lautrup, A. Peterman, E. de Rafael, Phys. Rept. 3 (1972) 193.

[24] C. Aubin, T. Blum, Phys. Rev. D 75 (2007) 114502; P. Boyle, L. Del Debbio, E. Kerrane, J. Zanotti, Phys. Rev. D 85 (2012) 074504; X. Feng, K. Jansen, M. Petschlies, D.B. Renner, Phys. Rev. Lett. 107 (2011) 081802; M. Della Morte, B. Jager, A. Juttner, H. Wittig, JHEP 1203 (2012) 055.

[25] T. Blum, M. Hayakawa, T. Izubuchi, PoS LATTICE 2012 (2012) 022.

[26] M. Steinhauser, Phys. Lett. B 429 (1998) 158.

[27] P.A. Baikov, K.G. Chetyrkin, J.H. Kuhn and C. Sturm, Nucl. Phys. B 867 (2013) 182; C. Sturm, Nucl. Phys. B 874 (2013) 698; P.A. Baikov, A. Maier, P. Marquard, Nucl. Phys. B 877 (2013) 647.

[28] F. Jegerlehner, in Proceedings of "Fifty years of electroweak physics: a symposium in honour of Professor Alberto Sirlin's 70th birthday", New York University, 27-28 October 2000, J. Phys. G 29 (2003) 101.

[29] S.L. Adler, Phys. Rev. D 10 (1974) 3714.

[30] S. Eidelman, F. Jegerlehner, A. L. Kataev, O. Veretin, Phys. Lett. B 454 (1999) 369.

[31] G. Balossini, C.M. Carloni Calame, G. Montagna, O. Nicrosini, F. Piccinini, Nucl. Phys. B 758 (2006) 227.

[32] S. Actis et al., Eur. Phys. J. C 66 (2010) 585.

[33] S. Eidelman, F. Jegerlehner, Z. Phys. C 67 (1995) 585; F. Jegerlehner, Nucl. Phys. Proc. Suppl. 181-182 (2008) 135.

[34] M. Bicer et al. [TLEP Design Study Working Group Collaboration], JHEP 1401 (2014) 164 [arXiv:1308.6176 [hep-ex]]. 
[35] G. Aarons et al. [ILC Collaboration], Physics at the ILC, arXiv:0709.1893 [hep-ph].

[36] K. Hagiwara, A.D. Martin, D. Nomura, T. Teubner, Phys. Lett. B 649 (2007) 173; Phys. Rev. D 69 (2004) 093003.

[37] G. Abbiendi et al. [OPAL collaboration], Eur. Phys. J. C14 (2000) 373-425.

[38] L. Gatignon, private communication.

[39] P. Abbon et al. [COMPASS Collaboration], Nucl. Instrum. Meth. A 577 (2007) 455

[40] Mathieu Perrin-Terrin for the NA62 Collaboration, Proceed. 24th IWVD, Santa Fe, June 2015, pp.016. 